\title{
Experimental Studies of a Neural Oscillator for Biped Locomotion with QRIO
}

Conference Paper in Proceedings - IEEE International Conference on Robotics and Automation · May 2005 DOI: 10.1109/ROBOT.2005.1570183 · Source: IEEE Xplore

CITATIONS

100
READS

65

4 authors, including:

G. Endo

Tokyo Institute of Technology

78 PUBLICATIONS 1,205 CITATIONS

SEE PROFILE

Some of the authors of this publication are also working on these related projects:

Project

CONTEST (COllaborative Network for Training in Electronic Skin Technology) View project

Project

EU-FoF Factory-in-a-Day View project

All content following this page was uploaded by G. Endo on 06 January 2014. 


\title{
Experimental Studies of a Neural Oscillator for Biped Locomotion with QRIO
}

\author{
Gen Endo*†, Jun Nakanishi ${ }^{\dagger \ddagger}$, Jun Morimoto ${ }^{\dagger \ddagger}$ and Gordon Cheng ${ }^{\dagger \ddagger}$ \\ * Sony Intelligence Dynamics Laboratories, Inc., Tokyo, Japan \\ $\dagger$ ATR Computational Neuroscience Laboratories, Kyoto, Japan \\ $\ddagger$ Computational Brain Project, ICORP, Japan Science and Technology Agency, Kyoto, Japan \\ gendo@idl.sony.co.jp, \{xmorimo,jun, gordon\}@atr.jp
}

\begin{abstract}
Recently, there has been a growing interest in biologically inspired biped locomotion control with Central Pattern Generator (CPG). However, few experimental attempts on real hardware 3D humanoid robots have yet been made. Our goal in this paper is to present our achievement of 3D biped locomotion using a neural oscillator applied to a humanoid robot, QRIO. We employ reduced number of neural oscillators as the CPG model, along with a task space Cartesian coordinate system and utilizing entrainment property to establish stable walking gait. We verify robustness against lateral perturbation, through numerical simulation of stepping motion in place along the lateral plane. We then implemented it on the QRIO. It could successfully cope with unknown $3 \mathrm{~mm}$ bump by autonomously adjusting its stepping period. Sagittal motion produced by a neural oscillator is introduced, and then overlapped with the lateral motion generator in realizing 3D biped locomotion on a QRIO humanoid robot.
\end{abstract}

Index Terms-Neural Oscillator; Central Pattern Generator(CPG); Biped Locomotion; QRIO;

\section{INTRODUCTION}

This paper presents our experimental studies on a neural oscillator for biped locomotion with a full-body humanoid robot. In our previous work [1], we explored neural oscillators as a Central Pattern Generator(CPG) which produces inherent rhythmic patterns, for a planar biped robot. In [1], we demonstrated that robust steady walking was achieved with a physical planar biped robot using the proposed method.

Our work is motivated by a growing interest in the studies of biologically inspired locomotion control using a neural oscillator as a CPG. Taga and his colleague successfully applied a neural oscillator controller as a CPG for an 8-link simulated planar biped model [2]. They demonstrated that stable bipedal locomotion is achieved with appropriate neural connections and biologically based sensory feedback pathways - the walking pattern was not explicitly pre-designed before execution, however it is self-organized through mutual interaction of the nervous system, musclo-skeletal system and environment. Kimura's quadruped robot demonstrated remarkable terrain adaptability by combining mechanical compliance, state machine, sensory reflexes and neural oscillators [3]. Their results suggest that there are possible ways to increase robustness against unknown perturbations and to generate

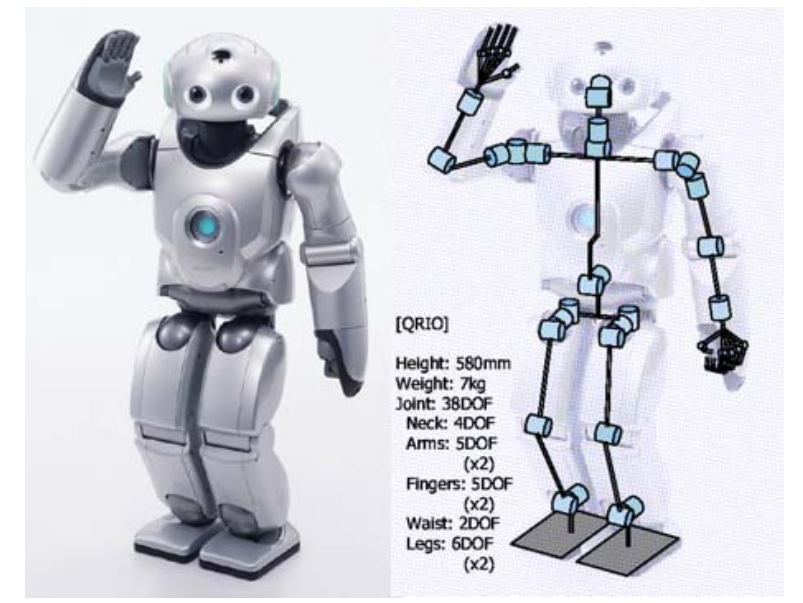

Fig. 1. Entertainment Robot QRIO (SDR-4X II)

more natural energy efficient motions exploiting passive dynamics by using the entrainment property of neural oscillators.

However, many of previous work on biped locomotion such as [2][4][5][6] were limited to simulation studies, and to our knowledge, there has been only few experimental application for a hardware three-dimensional (3D) humanoid robot [7] largely due to hardware limitations, difficulty in parameter tuning and large modelling error between simulations and experiments.

Our goal in this research is to achieve 3D biped locomotion using a neural oscillator with a full-body humanoid robot QRIO (SDR-4X II, see Fig. 1). To simplify the oscillator connections and feedback pathways, we propose an idea of allocating neural oscillators in a task space coordinate system. The advantage of the proposed arrangement is not only to significantly reduce the number of open parameters in the neural oscillator compared to the case where oscillators are allocated to each joint, but also to make it much easier to design effective feedback pathways to generate stable limit cycle.

This paper is organized as follows: In Section II, basic properties of neural oscillators and their arrangement for CPG are discussed. We design sensory feedback pathways to the neural oscillators to maintain balance of the body during locomotion motivated by biological observations. 
In Section III, we empirically verify the robustness of the proposed architecture by numerical simulations and hardware implementations. We demonstrate steady walking motion, robustness against lateral perturbation and an augmented example of biped locomotion in arbitrary directions following an operator's command. In Section IV, we will present summary of our results and future work.

\section{Proposed Control Architecture}

\section{A. Neural Oscillator Model}

We use the neural oscillator model proposed by Matsuoka [8], which is widely used as a CPG in robotic applications [3][9]:

$$
\begin{aligned}
\tau_{1} \dot{u}_{1} & =c-u_{1}-\beta v_{1}-\gamma\left[u_{2}\right]^{+}-\sum h_{j}\left[g_{j}\right]^{+} \\
\tau_{2} \dot{v}_{1} & =\left[u_{1}\right]^{+}-v_{1} \\
\tau_{1} \dot{u}_{2} & =c-u_{2}-\beta v_{2}-\gamma\left[u_{1}\right]^{+}-\sum h_{j}\left[g_{j}\right]^{-} \\
\tau_{2} \dot{v}_{2} & =\left[u_{2}\right]^{+}-v_{2} \\
q & =\left[u_{1}\right]^{+}-\left[u_{2}\right]^{+} \\
{[x]^{+} } & \stackrel{\text { def }}{=} \max (0, x), \quad[x]^{-} \stackrel{\text { def }}{=} \min (0, x)
\end{aligned}
$$

where $u_{1}, u_{2}, v_{1}$ and $v_{2}$ are internal states, $\beta$ and $\gamma$ are constants, $g_{j}$ is an input, and $q$ is an output of the oscillator. Time constants $\tau_{1}$ and $\tau_{2}$ characterize the output wave shape and its frequency, and a tonic excitation $c$ modulates the output amplitude.

Previously, properties of the neural oscillator model above such as the relationship between the parameters and the oscillator output has been numerically explored in [9] mainly addressing the issue of achieving stable oscillation with external feedback signals. It is demonstrated that phase difference between the periodic input signal $g_{j}$ and the output $q$ is tightly locked through entrainment if the amplitude of $g_{j}$ is large enough and its frequency is close to the oscillator's natural frequency.

In addition to the entrainment property mentioned above, our numerical studies suggest that it is possible to suppress
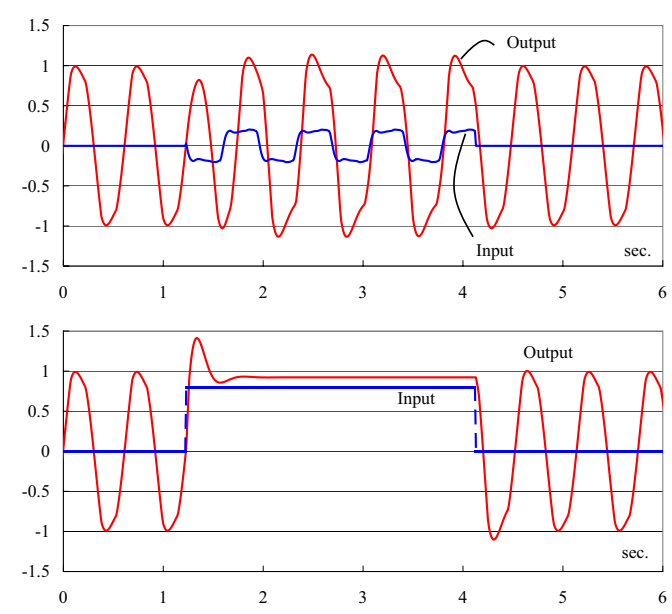

Fig. 2. Basic property of a neural oscillator (top: entrainment property, bottom: suppression of oscillation by a large constant input) the oscillation when an extremely large input signal is applied to the oscillator. Fig. 2 (top) illustrates an example of the entrainment property of the oscillator with a periodic input, and Fig. 2 (bottom) demonstrates that oscillation can be stopped with a large constant input signal to the oscillator. (See appendix for the parameters used in the neural oscillator model.) In this paper, we will exploit this additional property to maintain balance for stepping motion in place by stopping oscillatory movement of the legs with biologically motivated feedback pathways when a large external perturbation is applied to the robot.

\section{B. CPG Arrangement}

In many of the previous applications of neural oscillators based locomotion studies, an oscillator is allocated at each joint and its output is used as a joint torque command to the robot [2][6]. With this approach, it is demonstrated the desired gait can be generated by coordinating the movement of multiple oscillators in a self-organizing manner [2][6]. However, it is difficult to adjust the parameters of all the oscillators and feedback pathways to achieve the desired behavior with the increase of the number of degrees of freedom of the robot.

In our initial work [1], we proposed a compass-like biped arrangement of the CPG which provides an intuitive way of understanding the motion of the robot. In practice, since precise joint torque control is quite difficult to perform on a hardware system, we employed position based control. We demonstrated that robust steady walking was achieved with a physical planar biped robot using the proposed CPG arrangement.

In this paper, we extend our previous approach to a $3 \mathrm{D}$ robot model that has an increased number of degrees of freedom. To simplify the problem, we propose a new oscillator arrangement with respect to the position of the tip of the leg in the Cartesian coordinate system, which is reasonably considered as the task coordinates for walking. We allocate only a small set of neural oscillators exploiting symmetry of the walking pattern between the legs. Fig. 3 illustrates the proposed oscillator arrangement. We only employ the total of two oscillators to control the leg movement. One oscillator is allocated to control the position of both legs $p_{L z}, p_{R z}$ along the $Z$ (vertical) direction in a

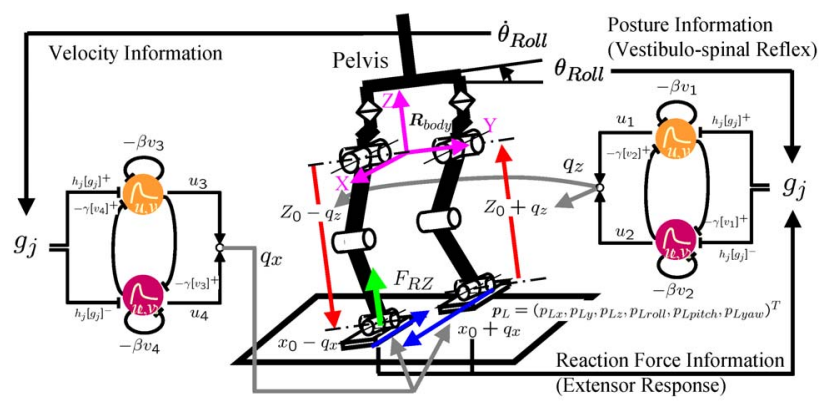

Fig. 3. Proposed control architecture using neural oscillators 
symmetrical manner with $\pi$ rad phase difference:

$$
\begin{aligned}
& p_{L z}=z_{0}-A_{z} q_{z} \\
& p_{R z}=z_{0}+A_{z} q_{z}
\end{aligned}
$$

where $q_{z}$ is the oscillator output for $Z$ direction, $z_{0}$ is an offset and $A_{z}$ is the amplitude scaling factor. Similarly, the other oscillator is allocated to control the position of both legs $p_{L x}, p_{R x}$ along the $X$ (forward) direction in the sagittal plane:

$$
\begin{aligned}
& p_{L x}=x_{0}-A_{x} q_{x} \\
& p_{R x}=x_{0}+A_{x} q_{x}
\end{aligned}
$$

where $q_{x}$ is the oscillator output for $X$ direction, $x_{0}$ is an offset and $A_{x}$ is the amplitude scaling factor. Each joint position of the leg is computed by using inverse kinematic calculation.

This arrangement significantly reduces the total number of open parameters in the neural oscillators and provides an easy way to find effective feedback pathways to achieve grobal entrainment because of the task space representation.

\section{Feedback Pathways}

In this section, we design sensory feedback pathways to the neural oscillators to maintain balance of the body during locomotion motivated by biological observations.

In biological systems, several reflexes were found in order to generate recovery momentum according to the inclination of the body by adjusting the leg length. For example, a decerebrate cat stomps stronger when vertical perturbation force applied to its plantar during extensor muscle activation. This reflex is called an Extensor Response [10]. It is generally known that vestibular system measures body's inclination and activates contralateral muscles to keep upper body stabilized. This is one of the basic posture control in human and called Vestibulospinal Reflex. Effectiveness of these feedback pathways was experimentally demonstrated with a hardware quadruped robot [3] to maintain the balance of the body when walking over unknown terrain. We incorporate these biologically motivated pathways into the CPG with our hardware humanoid robot to empirically investigate their effectiveness in biped locomotion. To our knowledge, no previous application of these ideas to a hardware biped robot can be found in the literature. Extensor Response and Vestibulospinal Reflex are formulated as follows:

1) Extensor Response:

$$
g_{E R}=\left(F_{L z}-F_{R z}\right) / m g
$$

where $F_{L z}, F_{R z}$ are left/right vertical reaction forces and feedback signal $g_{E R}$ is normalized by total body weight $m g$.

2) Vestibulo-spinal Reflex:

$$
g_{V S R}=\theta_{\text {roll }}
$$

A feedback pathway $g_{V S R}$ is introduced to extend leg length according to the body roll inclination $\theta_{\text {roll }}$.
These reflexes are incorporated into the neural oscillator controlling the vertical leg movement.

Furthermore, we introduce a feedback pathway to the oscillator controlling the horizontal leg movement, $q_{x}$, in the sagittal plane to achieve walking motion with the desired phase difference against $q_{z}$. As illustrated in Fig. 4, when the robot is walking forward, the leg trajectory with respect to the body coordinates in the sagittal plane can be roughly approximated by the shape of an ellipsoid. Suppose the output trajectories of the oscillators can be approximated as $p_{L x}=A_{x} \cos \left(\omega t+\alpha_{x}\right)$ and $p_{L z}=$ $A_{z} \cos \left(\omega t+\alpha_{z}\right)$, respectively. Then, to form the ellipsoidal trajectory on the $\mathrm{X}-\mathrm{Z}$ plane, $p_{L x}$ and $p_{L z}$ need to satisfy the relationship $p_{L x}=A_{x} \cos \phi$ and $p_{L z}=A_{z} \sin \phi$, where $\phi$ is the angle defined in Fig. 4. Thus, the desired phase difference between $q_{x}$ and $q_{z}$ should be $\alpha_{x}-\alpha_{z}=\pi / 2$.

Empirically, we found that $q_{z}$ and pelvis rolling angle $\theta_{\text {roll }}$ are almost in phase. Roughly speaking, differentiation of $\theta_{\text {roll }}$ has $\pi / 2$ phase difference with respect to $\theta_{\text {roll }}$ because differentiation of the sine wave is a cosine wave. From this observation, we can derive a signal in phase with $q_{x}$ using the pelvis rolling velocity signal $\dot{\theta}_{\text {roll }}$, which can be obtained from internal sensors of the robot.

3) Sagittal motion feedback:

$$
g_{x}=-\dot{\theta}_{\text {roll }}
$$

\section{EMPIRICAL VERIFICATION}

In this section, we empirically verify the proposed architecture using a dynamics simulator as well as a QRIO humanoid robot.

\section{A. Straight Walking}

Figures 5-7 show the the simulation results of straight walking. In Fig. 5, the time courses of $q_{x}, q_{z}$ and their
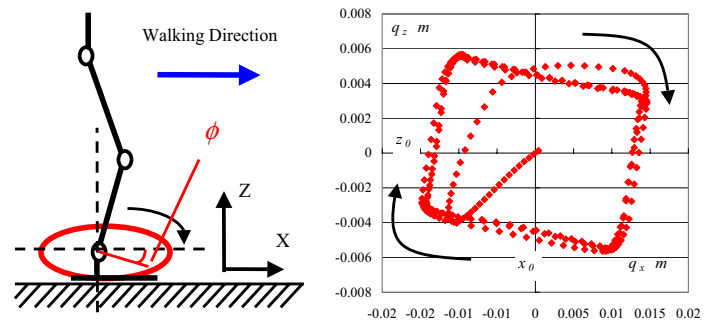

Fig. 4. Leg trajectory in the sagittal plane for forward walking (left: ellipsoidal trajectory, right: figure is plotted every $0.01 \mathrm{sec}$ for $3.0 \mathrm{sec}$. $A_{x}=0.015 \mathrm{~m}, A_{z}=0.005 \mathrm{~m}, \mathrm{~T}=0.8 \mathrm{~s}$.)

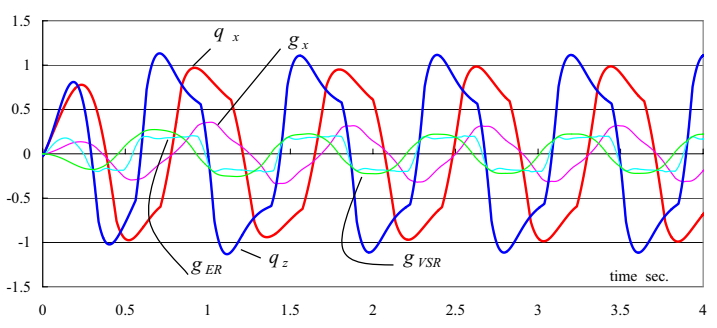

Fig. 5. $q_{x}, q_{z}$ entrainment and their feedback in the straight walking. 


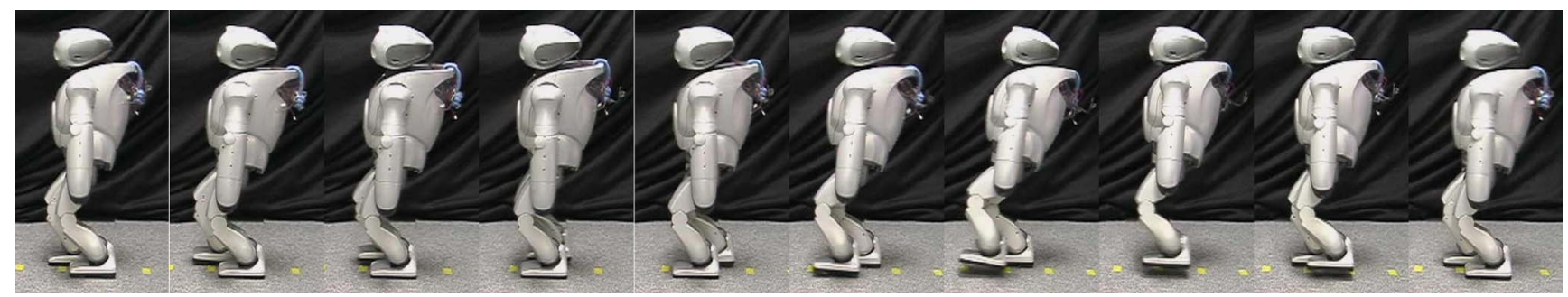

Fig. 6. Snapshots of straight steady walking $\left(A_{x}=0.030 \mathrm{~m}, A_{z}=0.005 \mathrm{~m}\right.$, Velocity $=0.12 \mathrm{~m} / \mathrm{s}$. Photos were captured every $0.1 \mathrm{sec}$. Yellow markers indicate every $0.1 \mathrm{~m}$ distance.)

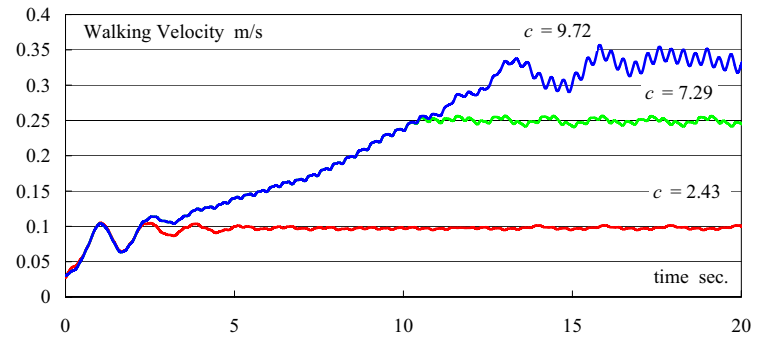

Fig. 7. Velocity control of straight walking gait using step length variation $\left(A_{x}=0.015 \mathrm{~m}, A_{z}=0.005 \mathrm{~m}, \mathrm{~T}=0.8 \mathrm{sec}\right)$.

feedback pathways are plotted. This result illustrates that entrainment of the neural oscillator with the feedback signals and the desired phase difference of $\pi / 2$ between $q_{x}$ and $q_{z}$ are achieved. Fig. 4 depicts the target leg trajectory on the $X Z$ plane for 3.0 sec starting from the initial standing posture suggesting a quick convergence to a periodic trajectory. Fig.7 shows the time course of walking velocity. With the proposed method, on-line modulation of average walking velocity is possible by gradually changing $c$ to vary the step length. We achieved walking with different velocities in the range of $0.0-0.33$ $\mathrm{m} / \mathrm{s}$ in forward locomotion (see Fig. 7). We also achieved backward walking by simply changing the sign of $A_{x}$.

We also implemented this steady walking control algorithm on QRIO, and achieved successful walking (see Fig. 6). We could modulate the walking velocity in the range of $0.0-0.2 \mathrm{~m} / \mathrm{s}$ with the step length variation.

\section{B. Stepping Motion in place}

In this section, we investigate robustness of a stepping motion in place against lateral perturbation by setting $A_{x}=0$. To obtain fundamental qualitative property, first we numerically confirm that natural robustness of sinusoidal stepping motion without feedback pathways according to the stepping period as a baseline. Secondly, we present the effectiveness of the proposed feedback pathways to the neural oscillator. Finally, we demonstrate autonomous adjustment of the stepping period using a hardware robot walking over bumped surfaces.

1) Natural Robustness: Here we drive the vertical leg movements by sinusoidal wave instead of a neural oscillator to investigate intrinsic stepping robustness. When the stepping motion converged on a stable limit cycle, constant perturbation force in the lateral positive direction was applied at the center of pelvis for $0.1 \mathrm{sec}$. We measured maximum allowable force without falling over. We

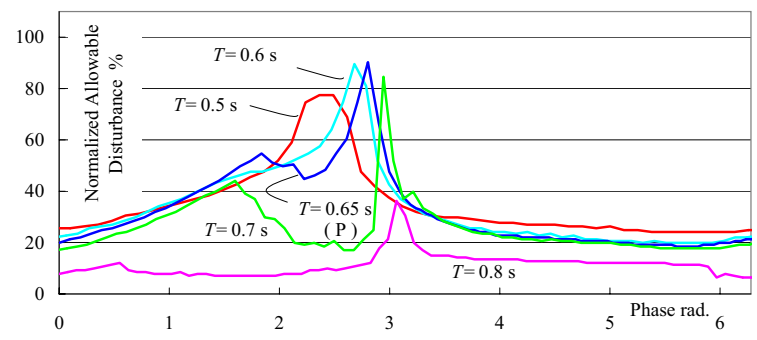

Fig. 8. Natural robustness of stepping motion in place using sinusoidal wave $\left(A_{z}=0.005 \mathrm{~m}\right)$.

regarded the perturbation force allowable when the robot kept stepping for $15 \mathrm{sec}$ after perturbation.

Fig. 8 illustrates the relationship between perturbation phase and force normalized by the stepping period and body weight respectively in numerical simulations. We define phase zero when the left leg touches the ground. Since stepping motion produces oscillatory change of angular momentum around the body roll axis, maximum perturbation force decreases when perturbation is applied in the direction of angular momentum increase. Similar tendency is observed in the case of $T=0.5-0.6$. However as point $(\mathrm{P})$ indicates, robustness rapidly decreases as the period becomes longer. We observed that the robot fell over very easily even with a small perturbation when the frequency of the stepping pattern is near the (roughly estimated) resonant frequency of the system. Presumably, this is because oscillation of the body is quickly amplified by resonance.

Fig. 8 suggests that shorter stepping period is desirable when we only consider inherent robustness of stepping motion. However, it draws heavier load for a hardware especially in touch down phase and requires larger energy consumption. Moreover, it is difficult to keep higher leg clearance due to the joint velocity limitation. If we can increase robustness near resonant frequency by using entrainment property of a neural oscillator, we can acquire more robust and energy efficient stepping motion.

2) Robustness with proposed feedback pathways: We compare the case where feedback pathways are introduced and not introduced to a neural oscillator for stepping motion in numerical simulations. Fig. 9 depicts a typical examples of the time course of pelvis roll angle $\theta_{\text {roll }}$ where perturbation is applied at the time of zero. While the robot falls over without sensory feedback to the neural oscillator, $\theta_{\text {roll }}$ with $g_{E R}$ feedback (gray line) converges for a certain 


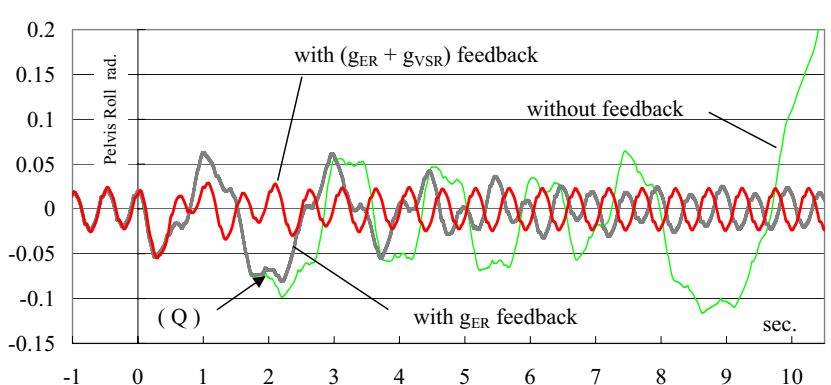

Fig. 9. Convergence difference according to feedback pathways

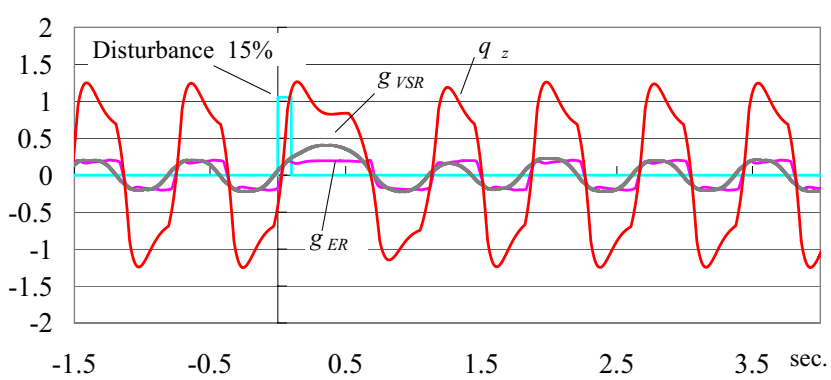

Fig. 10. Period extension of $q_{z}$ with vestibulo-spinal reflex

distant time. However, we cannot observe significant difference during the time 0.0-2.0 between the case where $g_{E R}$ is fed back and not fed back. Noticeably, at point (Q) indicated in Fig. 9, $q_{z}$ decreases supporting leg length. This motion decreases potential barrier preventing falling from occurring. Therefore, we introduce significantly large feedback gain for $g_{V S R}$ to suppress oscillation where $\theta_{\text {roll }}$ is large. When oscillation is stopped, passive dynamics play a role of recovery momentum generation. Fig. 9 (bold line) shows the faster entrainment of $\left(g_{E R}+g_{V S R}\right)$ than $g_{E R}$ only. Fig. 10 shows stepping period modulation with $\left(g_{E R}+g_{V S R}\right)$ feedback according to the body inclination. We can see the period modulation due to the feedback. Generally, it is difficult to entrain such a long period input using fixed time constants $\tau_{1}, \tau_{2}$. Thus some approaches adjust these parameters like phase lock loop manner to enlarge a basin of attraction [11]. In this paper, we utilize suppression of oscillation not to increase additional open parameters.

We investigated robustness against perturbation as well as the sinusoidal case. Entrainment property remarkably improved robustness compared with the case without feedback (Fig. 11). Even if stepping frequency was close to the resonant frequency, sensory feedback pathways still maintained as much robust as shorter period stepping of sinusoidal wave. This result suggests that a neural oscillator with appropriate sensory feedback can generate robust and energy efficient stepping motion.

Finally, we examined more realistic perturbation by performing the stepping motion on uneven surfaces. When the left leg was in swing phase, the ground height was altered for the next step (Fig. 12). In the case of $T=0.8$ without feedback, maximum allowable height were less than $2 \mathrm{~mm}$
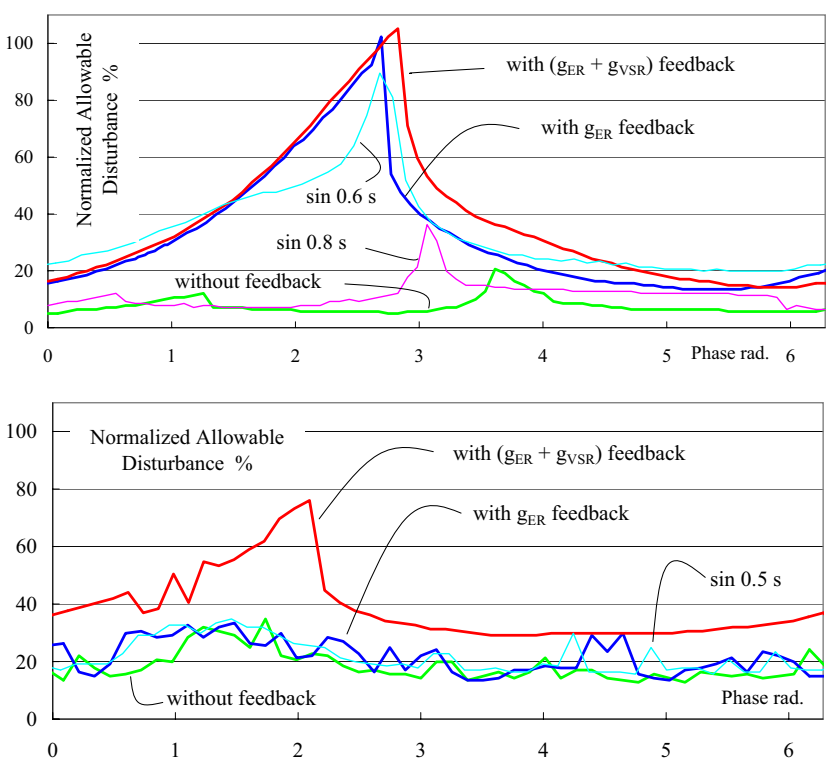

Fig. 11. Maximum allowable disturbance (top: $T=0.8 \mathrm{sec}, A_{z}=0.005 \mathrm{~m}$, bottom: $T=0.5 \mathrm{sec}, A_{z}=0.011 \mathrm{~m}$ )
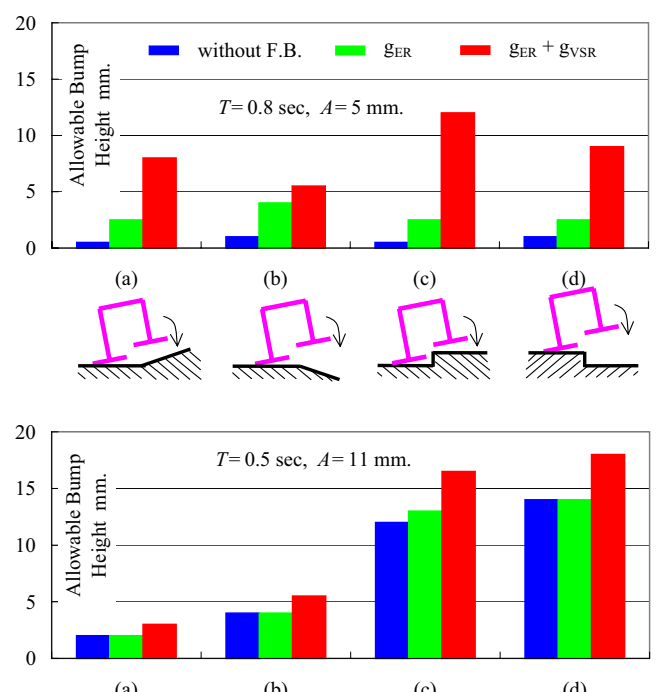

(a)

(b)

(c)

(d)

Fig. 12. Robustness against bump disturbance (top: $T=0.8 \mathrm{sec}, A_{z}=0.005$ m. bottom: $\left.T=0.5 \mathrm{sec}, A_{z}=0.011 \mathrm{~m}\right)$.

in the all situation(a)-(d), showing quite fragile limit cycle. On the contrary, $\left(g_{E R}+g_{V S R}\right)$ successfully increased it as well as $T=0.5 \mathrm{sec}$ condition, demonstrating effectiveness of extensor response and vestibulo-spinal reflex.

3) Hardware Evaluation: We investigated the effectiveness of feedback pathways to a neural oscillator at $T=$ $0.8 \mathrm{sec}$ using QRIO. Stepping motion without feedback was compared with the case where the proposed feedback pathways were introduced.

The robot could not continue a stepping motion on carpet floor without feedback. An amplitude of pelvis oscillation gradually increased and finally the robot fell over within 


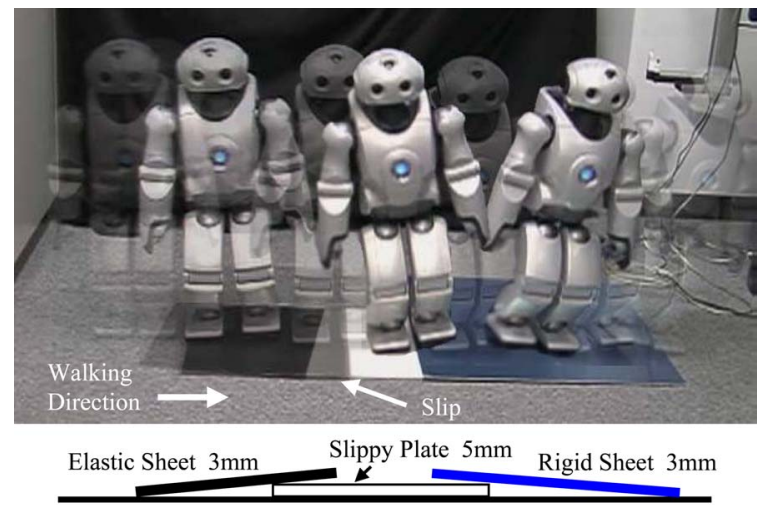

Fig. 13. Lateral walking experiment on bumped surfaces

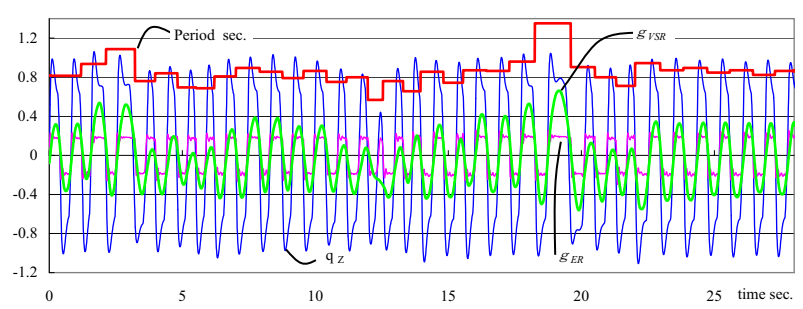

Fig. 14. Time courses of the stepping period, $q_{Z}, g_{E R}$ and $g_{V S R}$

about 20 steps.

In contrast, $\left(g_{E R}+g_{V S R}\right)$ feedback pathways demonstrated a highly stable stepping motion which could cope with $3 \mathrm{~mm}$ of (a), $7 \mathrm{~mm}$ of (c) height deviations, respectively (see Fig. 12 in the middle row for the situations). Although, these values were less than simulated values due to sensing delay caused by integration of angular rate sensor to obtain the body rolling angle $\theta_{\text {roll }},\left(g_{E R}+g_{V S R}\right)$ feedback pathways considerably improved robustness against perturbation.

We also performed a walking experiment over unknown terrain in the lateral direction with state-machine controller (we will mention in the following section). The condition of walking surface changed as it walked due to seesawing or slipping because the sheets were not rigidly connected. Nevertheless, a neural oscillator autonomously adjusted its period in the wide range and successfully made the robot walk (Fig. 13).

\section{Walking under user command}

We show an augmented example of above mentioned framework to demonstrate enough capability to extend a state-machine controller. We can design explicit trajectory in the horizontal $X Y$ direction because the stepping motion is generated by the vertical oscillatory movement. Since basic stepping movement in the lateral plane is already realized, walking motion will be possible if the legs are moved with the appropriate timing. Here, we assume quasistatic movements and only consider kinematic constraints of the body and the stance leg. State-machine controllers in the $X$ direction are defined as follows:

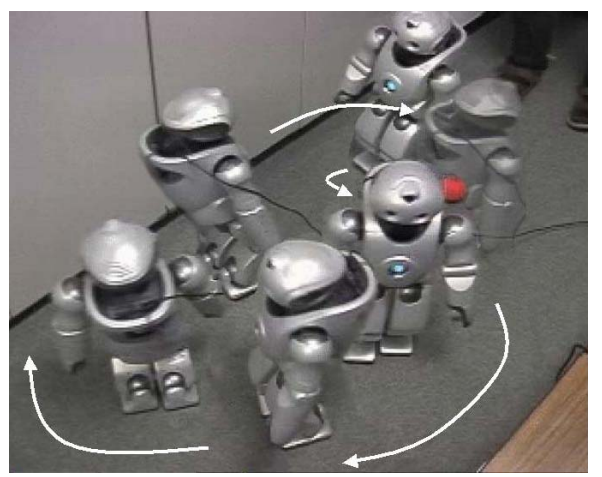

Fig. 15. Manuvoring experiment using combination of CPG and statemachine controller

- Stance Phase

$$
\begin{aligned}
& p_{L x}^{\prime}=p_{L x}^{t d}-\left(t-t^{t d}\right) /(T / 2) \cdot x_{\text {stride }} \\
& p_{L x}=\max \left(p_{L x}^{\prime},-x_{\text {stride }} / 2\right)
\end{aligned}
$$

- Swing Phase

$$
\begin{aligned}
& p_{L x}^{\prime}=p_{L x}^{l o}+\left(t-t^{l o}\right) /(T / 2) \cdot x_{\text {stride }} \\
& p_{L x}=\min \left(p_{L x}^{\prime}, x_{\text {stride }} / 2\right)
\end{aligned}
$$

where $t, T, x_{\text {stride }}$ represent time, period and commanded walking stride, respectively. $p_{L x}^{t d}, t^{t d}, p_{L x}^{l o}, t^{l o}$ denote left leg touch down position and its time, lift off position and its time, respectively. We implemented the same controller for the right leg control. Although there is no explicit feedback which stabilizes movement in the sagittal plane, a limit cycle is consequently established due to foot contact trigger for state transitions. Walking velocity is controlled by adjusting a step stride $x_{\text {stride }}$ as well as the case of neural oscillator based controller. We can apply the same controller in the $Y$ direction to achieve a side walk. In this case, a sideway stepping causes disturbance in the lateral direction. However, entrainment property can handles it in a certain amount of range (Fig. 13).

A circular walking is attained by combining the abovementioned X-Y direction stepping along with a particular circular arc, modulating the left/right step length according to the circular radius. The leg yaw rotation is also controlled by the function of X-Y position.

In addition, gait initiation and termination are achieved by altering neural oscillator amplitude modified by a tonic parameter $c$ in Eq. (1). If $c$ gradually decreases, then a stepping motion is terminated.

The above framework was implemented on the hardware and a manuevouring experiment was demonstrated. The robot could interactively follow directive given by an operator (Fig. 15).

This result indicates entrainment property with appropriate feedback pathways has enough capability for interactive locomotion. 


\section{CONCLUSion}

In this paper, we proposed a basic framework for biped locomotion control of a 3D hardware biped robot using a neural oscillator. A new CPG arrangement and biologically inspired feedback pathways to the neural oscillators were introduced. We implemented the proposed method in numerical simulations and the humanoid robot QRIO. First, we demonstrated that straight walking with different velocities was achieved by changing the tonic input to the neural oscillators. Then, we investigated the robustness of stepping motion in the lateral plane. The simulation and experimental results suggest that the proposed feedback pathways play contribute to robustness against external perturbations and environmental changes. Finally, we presented an example of augmentation of the proposed framework with a state-machine controller to achieve walking under user command. We were able to maneuver QRIO by changing the walking direction and velocity through teleoperation.

As the results in this paper suggests, various walking behavior can be generated with this simple approach. We believe that this paper is the first to experimentally implement neural oscillators on a 3D hardware humanoid robot achieving biped locomotion in the physical environment.

In this paper, we manually tuned the neural oscillator parameters and used constant feedback coefficients empirically obtained through numerical simulations and hardware implementation. We would like to address the issues of optimizing these open parameters with a learning framework. Our recent work address foot placement learning [12] using reinforcement learning, energy efficient robust walking using differential dynamic programming [13], and learning an appropriate feedback controller to the CPG with a policy gradient [14] for a simplified planar biped robot. In the future, we are interested in extending these approaches to high dimensional systems and experimental implementation to a 3D hardware biped robot.

\section{ACKNOWLEDGEMENT}

We would like to thank all the persons concerned in QRIO's development for supporting this research. We would like to thank Seiichi Miyakoshi of the Digital Human Research Center, AIST, Japan for productive and invaluable discussions.

\section{REFERENCES}

[1] G. Endo, J. Nakanishi, J. Morimoto, and G. Cheng, "An empirical exploration of a neural oscillator for biped locomotion control," in IEEE International Conference on Robotics and Automation, 2004, pp. 3036-3042.

[2] G. Taga, "A model of the neuro-musculo-skeletal system for human locomotion I. emergence of basic gait," Biological Cybernetics, vol. 73, pp. 97-111, 1995.

[3] H. Kimura, Y. Fukuoka, and A. H. Cohen, "Biologically inspired adaptive dynamic walking of a quadruped robot," in 8th International Conference on the Simulation of Adaptive Behavior, 2003, pp. 201-210.

[4] K. Hase and N. Yamazaki, "A self-organizing model to imitate human development for autonomous bipedal walking," in 6th International Symposium on Computer Simulation in Biomechanics, 1997, pp. 9-12.
[5] M. Sato, Y. Nakamura, and S. Ishii, "Reinforcement learning for biped locomotion," in International Conference on Artificial Neural Networks, 2002, pp. 777-782.

[6] A. Ishiguro, A. Fujii, and P. E. Hotz, "Neuromodulated control of bipedal locomotion using a polymorphic cpg circuit," Adaptive Behavior, vol. 11, no. 1, pp. 7-17, 2003.

[7] M. Matsuura, K. Ishimura, and M. Wada, "Emergence of biped robot locomotion through neural entrainment," Journal of Robotics Society of Japan, vol. 22, no. 3, pp. 56-62, 2004, in Japanese.

[8] K. Matsuoka, "Sustained oscillations generated by mutually inhibiting neurons with adaptation," Biological Cybernetics, vol. 52, pp. 345-353, 1985.

[9] M. Williamson, "Neural control of rhythmic arm movements," Neural Networks, vol. 11, no. 7-8, pp. 1379-1394, 1998.

[10] A. H. Cohen and D. L. Boothe, "Sensorimotor interactions during locomotion: Principles derived from biological systems," Autonomous Robotics, vol. 7, no. 3, pp. 239-245, 1999.

[11] S. Kotosaka and S. Schaal, "Synchronized robot drumming by neural oscillator," Journal of the Robotics Society of Japan, vol. 19, no. 1, pp. 116-123, 2001.

[12] J. Morimoto, G. Cheng, C. G. Atkeson, and G. Zeglin, "A simple reinforcement learning algorithm for biped walking," in IEEE International Conference on Robotics and Automation, 2004, pp. 3036-3042.

[13] J. Morimoto, G. Zeglin, and C. G. Atkeson, "Minimax differential dynamic programming: Application to a biped walking robot," in IEEE International Conference on Intelligent Robots and Systems, 2003, pp. 1927-1932.

[14] T. Matsubara, J. Morimoto, J. Nakanishi, and K. Doya, "Learning feedback pathways in CPG with policy gradient for biped locomotion," in IEEE International Conference on Robotics and Automation, 2005, (in preparation).

\section{APPENDIX}

Common parameters for all neural oscillators. Initial internal variables are set to encourage faster convergence. They are captured at $(q=0, \dot{q}>0)$ where a stable oscillation is established.

\begin{tabular}{|l||c|c|}
\hline \multicolumn{1}{|c||}{ Description } & Symbol & Value \\
\hline \hline Time constant & $\tau_{1}$ & $0.1122 \cdot T$ \\
\hline Time constant ratio & $\tau_{1} / \tau_{2}$ & 0.8 \\
\hline Mutual inhibition & $\gamma$ & 2.0 \\
\hline Adaptation constant & $\beta$ & 2.5 \\
\hline Tonic excitation & $c$ & 2.43 \\
\hline \hline Initial internal variable & $u_{1}^{\text {initial }}$ & 0.488488 \\
\hline Initial internal variable & $v_{1}^{\text {initial }}$ & 0.203274 \\
\hline Initial internal variable & $u_{2}^{\text {initial }}$ & 0.389224 \\
\hline Initial internal variable & $v_{2}^{\text {initial }}$ & 0.674099 \\
\hline
\end{tabular}

Feedback coefficients:

\begin{tabular}{|l||c|c|}
\hline \multicolumn{1}{|c||}{ Description } & Symbol & Value \\
\hline \hline Extensor Response & $h_{E F}$ & 0.2 \\
\hline Vestibulo-spinal Reflex & $h_{V S R}$ & 4.0 \\
\hline Sagittal motion feedback & $h_{x}$ & 0.2 \\
\hline
\end{tabular}

These symbols are abbreviated in figures to indicate feedback signals. 